\title{
Dispersive MHD waves and alfvenons in charge non-neutral plasmas
}

\author{
K. Stasiewicz ${ }^{1,2}$ and J. Ekeberg ${ }^{1}$ \\ ${ }^{1}$ Swedish Institute of Space Physics, Uppsala and Kiruna, Sweden \\ ${ }^{2}$ Space Research Centre, Polish Academy of Sciences, Warsaw, Poland
}

Received: 10 March 2008 - Revised: 13 June 2008 - Accepted: 12 July 2008 - Published: 8 August 2008

\begin{abstract}
Dispersive properties of linear and nonlinear MHD waves, including shear, kinetic, electron inertial Alfvén, and slow and fast magnetosonic waves are analyzed using both analytical expansions and a novel technique of dispersion diagrams. The analysis is extended to explicitly include space charge effects in non-neutral plasmas. Nonlinear soliton solutions, here called alfvenons, are found to represent either convergent or divergent electric field structures with electric potentials and spatial dimensions similar to those observed by satellites in auroral regions. Similar solitary structures are postulated to be created in the solar corona, where fast alfvenons can provide acceleration of electrons to hundreds of $\mathrm{keV}$ during flares. Slow alfvenons driven by chromospheric convection produce positive potentials that can account for the acceleration of solar wind ions to $300-800 \mathrm{~km} / \mathrm{s}$. New results are discussed in the context of observations and other theoretical models for nonlinear Alfvén waves in space plasmas.
\end{abstract}

\section{Introduction}

In a recent review, Paschmann et al. (2002) provide a long list of problems in auroral physics that still remain unsolved. Similarly, there is an ongoing debate on some 20 mechanisms proposed for the acceleration of plasma in the solar corona (Aschwanden, 2004). While observations in the solar corona can be made only remotely, which strongly limits spatial resolution, there are many detailed measurements in the magnetosphere. Both optical and in situ measurements show that acceleration of auroral particles occur on two distinctive spatial scales. The large scale $\sim 50 \mathrm{~km}$ is associated with inverted-V type electron acceleration, which

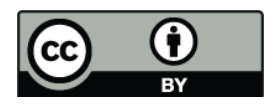

Correspondence to: K. Stasiewicz (k.stasiewicz@irfu.se) is pictured in the form of convergent electric field structures that would accelerate electrons into and ions out of the ionosphere (Gurnett, 1972; Mozer et al., 1980). The small scales correspond to thin arcs with thickness $\sim 1 \mathrm{~km}$, which can be associated with alfvénic structures on electron inertial scale length (Stasiewicz et al., 2000a).

In addition to convergent electric field structures found in regions with upward magnetic field-aligned currents, there are observations of divergent structures, which occur in downward current regions, and could be associated with void, or black aurora (Marklund et al., 1994; Carlson et al., 1998a; Ergun et al., 1998; Marklund et al., 2001). The key issues of auroral research concern the understanding how the negative and positive electric potential structures are formed, which driver provides the required energy, what determines their spatial scales, their electric polarization, and how the parallel electric fields within these structures are supported.

In this paper we shall present a unified view providing explanations for the major issues of auroral physics: (i) the presence of a wide range of spatial scales in auroral structures, (ii) the creation of negative and positive electric field structures, (iii) mechanisms supporting parallel electric fields, and (iv) drivers of auroral activity and electron acceleration. The model is universal and applicable both to acceleration of electrons producing aurora in the magnetosphere, and to the generation of solar wind and acceleration of particles producing X-ray emissions during solar flares.

Briefly, the model is based on nonlinear wave solutions of two-fluid equations. Alfvénic soliton solutions have been studied extensively using Derivative Nonlinear Schrödinger (DNSL) equation (Hada et al., 1989; Medvedev and Diamond, 1996; Mjølhus and Wyller, 1988; Nocera and Buti, 1996; Spangler, 1990; Buti, 1999; Passot et al., 2005), or complete one- or two-fluid equations (McKenzie et al., 2004; Dubinin et al., 2003, 2005; Sauer et al., 2003). However, until recently it has not been realized that soliton solutions represent bipolar electric field structures with either positive

Published by Copernicus Publications on behalf of the European Geosciences Union and the American Geophysical Union. 
potential (divergent electric field), or negative potential (convergent electric field). These solitary electric field structures have been numerically modelled by Stasiewicz (2006, 2007); Stasiewicz and Ekeberg (2008), who found that electric potentials of several $\mathrm{kV}$ in aurora, and a few hundred $\mathrm{kV}$ in the solar corona can be created on spatial scales ranging from the electron inertial length, $\lambda_{e}=c / \omega_{p e}$, to a few ion inertial lengths, $\lambda_{i}=c / \omega_{p i}$, where $\omega_{p e}, \omega_{p i}$ denote the electron and ion plasma frequencies, and $c$ is the speed of light. The electron, $\lambda_{e}$ and ion scale $\lambda_{i}=\lambda_{e} \sqrt{m_{i} / m_{e}}$ determine two scales of solitary structures responsible for the acceleration of auroral electrons. Note that $\lambda_{e} \sim 1 \mathrm{~km}$ and $\lambda_{i} \sim 50 \mathrm{~km}$ in a proton plasma with the density of $N \sim 20 \mathrm{~cm}^{-3}$, which could correspond to conditions in the auroral acceleration region at altitudes of $1 R_{E}$.

The creation of large amplitude electric field structures implies that space charge effects may play important role in particle acceleration and should be explicitly included in the governing equations. This issue has been recently highlighted by a controversy between Verheest $(2007 \mathrm{a}, \mathrm{b})$ and McKenzie et al. (2007) on the existence of solitary waves in charge neutral plasmas.

We shall also make brief review of the progress in the theory and applications of nonlinear waves to acceleration of particles and comment on confusing nomenclature and some misconceptions present in the literature on dispersive Alfvén waves. For example, the universal importance of the ion inertial scale $\lambda_{i}$ for all MHD waves has not been recognized, and focus has been on electron inertia $\lambda_{e}$, which is relevant only for propagation perpendicular to the magnetic field $\mathbf{B}$ in very cold plasmas $\left(\beta<m_{e} / m_{i}\right)$.

It is usually thought that nonlinear waves represent a sort of sine waves, but with larger amplitudes. This is not the case, because solitary wave solutions occupy regions in the parameter space different from sinusoidal waves and represent separate nonlinear entities. We shall also point out that many published models of nonlinear Alfvén waves are questionable, because they are based on equations that impose artificial restrictions, e.g. a linear magnetic polarization, or electrons assumed to move only parallel while ions only perpendicular to $\mathbf{B}$.

The presence of a perpendicular potential drop implies the creation of a parallel potential of the same magnitude, and vice versa. A long lasting debate on the existence of electric fields parallel to $\mathbf{B}$ is bounded by two extreme positions: Some authors believe that parallel electric fields either do not exist, or cannot be understood in the frame of fluid equations. On the opposite side, there are authors who claim that parallel electric fields can be explained by ideal MHD equations (Tsiklauri, 2006). In Sect. 3, we show that the well known, "traditional" fluid equations in fact describe four mechanisms that contribute to the parallel electric field.

\section{Basic observations and measurements}

The properties of dispersive Alfvén waves in the electron inertial limit have been investigated with the Large Plasma Device at UCLA (Gekelman et al., 2008) in many experimental setups and beta regimes. Gekelman et al. (2000) made measurements of the propagation of waves with ion temperatures and drifts measured using laser induced fluorescence, a diagnostic that was later improved by Palmer et al. (2005). Dispersive properties of waves at the critical $\beta \approx m_{e} / m_{i}$, corresponding to the transition between the regimes dominated by, on one hand, electron inertia and, on the other hand, kinetic effects, were studied by Kletzing et al. (2003) and Vincena et al. (2004). Here, $\beta=\beta_{i}+\beta_{e}$ denotes the ratio of plasma and magnetic field pressures. These experiments enable repetitive, detailed measurements of wave properties and also serve as tests of various theories and approximations.

In space physics, the high resolution measurements of Freja (Lundin et al., 1998) and FAST (Carlson et al., 1998b) have generated great interest in the study of dispersive Alfvén waves in the auroral ionosphere. Freja observations of spatially localized strong electromagnetic pulses were interpreted first as signatures of solitary kinetic Alfvén waves (SKAW) by Louarn et al. (1994) and Wahlund et al. (1994). The terminology used by these authors was unfortunate, because measurements made by Freja were done in a predominantly low beta environment, $\beta<m_{e} / m_{i}$, where kinetic Alfvén waves (KAW) do not exist. The observations were related to intense current structures $j_{\|} \sim 100 \mu \mathrm{A} \mathrm{m}^{-2}$ on the electron inertial scale (Stasiewicz et al., 1997, 1998; Ivchenko and Marklund, 2002). The use of KAW terminology to describe electron inertial structures caused considerable confusion among other authors who continued to mix kinetic, finite-beta effects with electron inertial effects.

Observations made by the POLAR spacecraft at distances 4-6 $R_{E}$ (Earth radii) have shown the presence of Poynting flux carried by Alfvén waves with magnitude sufficient to drive auroral phenomena (Wygant et al., 2000; Keiling et al., 2000; Keiling et al., 2002). These results were challenged by Janhunen et al. (2006) who claimed that parallel Poynting flux measured at radial distances $4-5 R_{E}$ is insufficient to account for auroral energization of electrons. Using FAST data, Chaston et al. $(2003,2005)$ investigated the width and brightness of auroral arcs and energy deposition by field-aligned electrons. They confirmed a typical thin structure thickness of $\sim 1 \mathrm{~km}$ and found an energy deposition of $\sim 10 \mathrm{~mW} / \mathrm{m}^{2}$.

Observations in the solar atmosphere reveal the presence of various modes of MHD waves (DeMoortel et al., 2000; Robbrecht et al., 2001). Outward propagating Alfvén waves in the solar corona have also been investigated using Faraday rotation fluctuations from the Helios spacecraft by Samoznaev et al. (2000). Observational evidence for the presence of MHD waves in the corona have been reviewed by Banerjee et al. (2007). A connection between Alfvén and slow mode 
waves, and sources of solar wind has recently been confirmed with Hinode data by Cirtain et al. (2007); Sakao et al. (2007).

\section{Acceleration by electric fields}

Starting with the equation for the Lorentz force acting on a particle with mass $m$, and charge $q$ in electromagnetic fields,

$m \frac{\mathrm{d} \mathbf{v}}{\mathrm{d} t}=q(\mathbf{E}+\mathbf{v} \times \mathbf{B})$,

and making the dot product with velocity $\mathbf{v}$, we see that charged particles can be heated or accelerated by the electric field only:

$\frac{\mathrm{d}}{\mathrm{d} t}\left(\frac{m v^{2}}{2}\right)=q E_{\|} v_{\|}+q \mathbf{E}_{\perp} \cdot \mathbf{v}_{\perp}$.

The magnetic field does not accelerate particles, but the timevarying field produces an electric field, $\nabla \times \mathbf{E}=-\partial \mathbf{B} / \partial t$ that can lead to (betatron) acceleration.

Acceleration of charged particles by parallel electric field $(\mathbf{E} \| \mathbf{B})$ is rather trivial, but there is a long debated problem concerning how the macroscopic $E_{\|}$can be maintained in view of the high parallel mobility of particles. Within the two-fluid model, the parallel electric field can easily be obtained from the generalized Ohm's law (e.g. Krall and Trivelpiece, 1973) as

$$
\begin{array}{r}
E_{\|}=\eta J_{\|}-\frac{\nabla_{\|} p_{\| e}}{N e}-\frac{\left(p_{\perp e}-p_{\| e}\right)}{N e} \frac{(\nabla B)_{\|}}{B} \\
+\frac{m_{e}}{N e^{2}}\left[\nabla \cdot\left(\mathbf{V} J_{\|}+\mathbf{J} V_{\|}\right)+\frac{\partial J_{\|}}{\partial t}\right],
\end{array}
$$

which describes four mechanisms supporting $E_{\|}$. These are: (i) classical or anomalous resistivity, $\eta$, (ii) the electron pressure gradient along the magnetic field, (iii) the magnetic mirror force acting on anisotropic electron pressures, and (iv) electron inertial effects. An additional mechanism for the parallel electric field can be provided by double layers (Charles, 2007), which are on smaller (Debye length) scale and require kinetic description. Phenomenologically, they can be included in Eq. (3) as a sort of anomalous resistivity.

The perpendicular electric field first induces the electric $\operatorname{drift} \mathbf{V}_{E}=\mathbf{E} \times \mathbf{B} / B^{2}$, which does not lead to heating. However, a sufficiently large gradient of the electric field would break the symmetry of Larmor orbits and lead to perpendicular energization. A necessary condition for this type of acceleration is (Cole, 1976)

$$
\frac{1}{\omega_{c} B}\left|\frac{\partial E_{x}}{\partial x}\right| \gtrsim 1
$$

which means that the scale size of the electric field gradient must be sufficiently small, $L \lesssim E_{x} / \omega_{c} B$, where $\omega_{c}=q B / m$ is the gyrofrequency. This is the basic mechanism for perpendicular ion heating in space plasmas that was invoked by a few authors (Balikhin et al., 1993; Mishin and Banaszkiewicz, 1998; Stasiewicz et al., 2000b; Voitenko and Goossens, 2004). Because of the mass dependence in Eq. (4), this criterium is first met by heavy ions, which is indeed observed in the magnetosphere and in the solar corona, and reported as preferential perpendicular heating of heavy ions (e.g. Kohl et al., 1997; Cranmer et al., 1999).

The electric field gradient implies charge non-neutrality $\delta N_{e}=\left|N_{e}-N_{i}\right|$ that can be determined from the Poisson's equation

$\varepsilon_{0} \nabla \cdot \boldsymbol{E}=e\left(N_{i}-N_{e}\right)$.

The condition (4) implies that ion energization on electric field gradients requires a charge non-neutrality of

$\frac{\delta N_{e}}{N} \gtrsim \frac{\omega_{c i}^{2}}{\omega_{p i}^{2}}=\frac{V_{A}^{2}}{c^{2}}$,

which is small in non-relativstic plasmas and justifies the use of quasi-neutrality, $N_{e} \approx N_{i}$, together with $d E_{x} / d x \neq 0$. In the solar corona, $V_{A}^{2} / c^{2}<10^{-4}$, but this parameter can be larger in auroral acceleration regions.

\section{Poynting flux and energy sources for particle acceler- ation}

Poynting fluxes associated with the convective motion of plasma, $\mathbf{V}_{E}$, in the terrestrial magnetosphere, the solar corona, and in astrophysical objects,

$\mathbf{S}=\frac{1}{\mu_{0}} \mathbf{E} \times \mathbf{B}=\mathbf{V}_{E} \frac{B^{2}}{\mu_{0}}$,

represent gigantic sources of power. Tapping a small portion of this perpendicular energy flux to feed a wave propagating obliquely to the magnetic field would provide electromagnetic power in the direction parallel to the magnetic field. This could explain the energy sources for many plasma acceleration processes observed in space.

Let us consider an MHD wave in the plasma rest frame of reference, with speed $\omega / k$ in the $x$ direction at an angle $\alpha$ to the background magnetic field $\mathbf{B}_{0}=B_{0}[\cos \alpha, 0, \sin \alpha]$. We assume that plasma parameters vary mainly along $x$, so that $\partial / \partial x \gg \partial / \partial y, \partial / \partial z$, and transverse gradients can be neglected. Under such an assumption, the electric and magnetic fields in the plasma frame are: $\mathbf{E}=\left[\delta E_{x}, \delta E_{y}, \delta E_{z}\right]$ and $\mathbf{B}=\left[B_{x 0}, \delta B_{y}, B_{z 0}+\delta B_{z}\right]$, where delta quantities represent wave perturbations of arbitrary amplitudes. The coupling between waves and plasma flows can be accomplished by the divergence of the Poynting flux, which in 1-D reads: $\nabla \cdot \mathbf{S}=\partial S_{x} / \partial x \neq 0$, where $\mu_{0} S_{x}=E_{y} B_{z}-E_{z} B_{y}$. The component of $\mathbf{S}$ along the $\mathbf{B}_{0}$ direction is given generally by

$\mu_{0} S_{\| 0}=\left(\sin \alpha \delta E_{x}-\cos \alpha \delta E_{z}\right) \delta B_{y}+\cos \alpha \delta E_{y} \delta B_{z}$,

where $\delta B_{y}$ and $\delta B_{z}$ correspond to alfvénic and magnetosonic polarizations, respectively. In case of sinusoidal waves, 
$\propto \exp (i k x-i \omega t)$, the Faraday's law $\nabla \times \mathbf{E}=-\partial \mathbf{B} / \partial t$ implies

$\delta E_{y}=(\omega / k) \delta B_{z}, \quad \delta E_{z}=-(\omega / k) \delta B_{y}$,

while the $x$ component can be obtained from the generalized Ohm's law (Eq. 30) as

$\frac{\delta E_{x}}{V_{A} B_{0}} \approx M \frac{\delta B_{y}}{B_{0}} \tan \alpha-\frac{\beta_{e} \lambda_{i}}{2 n} \frac{\partial}{\partial x}\left(\frac{p_{e}}{p_{e 0}}\right)$.

Here, $n=N / N_{0}, V_{A}=B_{0}\left(\mu_{0} N_{0} m_{i}\right)^{-1 / 2}$ is the Alfvén speed, $M=\omega / k V_{A}$ is the Alfvén Mach number, $p_{e}$ is the electron pressure, $\beta_{e}$ is the ratio of electron and magnetic field pressures, and $\lambda_{i}=V_{A} / \omega_{c i}$ is the ion inertial length. A small term proportional to $m_{e} / m_{i}$, the electron to ion mass ratio, is neglected in Eq. (10). It is easily verified that the parallel electric field, $\mathbf{E} \cdot \mathbf{B} / B$, contains only the electron pressure term, consistent with Eq. (3). Note also that the electric field $E_{x}$ is invariant in all coordinate systems moving along $x$. The Poynting flux in the plasma frame is then

$$
\frac{S_{\| 0}}{S_{A}}=M_{\|}\left(\frac{\delta B_{y}^{2}}{B_{0}^{2}}+\cos ^{2} \alpha \frac{\delta B_{z}^{2}}{B_{0}^{2}}\right)-\sin \alpha \frac{\beta_{e} \lambda_{i}}{2 n} \frac{\partial}{\partial x}\left(\frac{p_{e}}{p_{e 0}}\right) \frac{\delta B_{y}}{B_{0}},
$$

in units of $S_{A}=V_{A} B_{0}^{2} / \mu_{0}$, with $M_{\|}=M / \cos \alpha$. Replacing magnetic perturbations by velocity perturbations as implied by the momentum equations, $\delta B_{y, z} B_{0}^{-1}=M_{\|} \delta V_{y, z} V_{A}^{-1}$, we find an equivalent form

$$
\begin{aligned}
\frac{S_{\| 0}}{N_{0} m_{i} V_{A}^{3}}= & M_{\|}^{3}\left(\frac{\delta V_{y}^{2}}{V_{A}^{2}}+\cos ^{2} \alpha \frac{\delta V_{z}^{2}}{V_{A}^{2}}\right) \\
& -M_{\|} \sin \alpha \frac{\beta_{e} \lambda_{i}}{2 n} \frac{\partial}{\partial x}\left(\frac{p_{e}}{p_{e} 0}\right) \frac{\delta V_{y}}{V_{A}},
\end{aligned}
$$

which implies that there are ion flows $\delta V_{y}, \delta V_{z}$ that by shaking magnetic field lines $\delta B_{y}, \delta B_{z}$ create parallel Poynting flux $S_{\| 0}$. For a particular mode, one has to use a suitable Mach number: $M=\cos \alpha$ for Alfvén modes, $M=1$ for fast magnetosonic waves, $M=(\gamma \beta / 2)^{1 / 2} \cos \alpha$ for slow magnetosonic waves, and $M=(\gamma \beta / 2)^{1 / 2}$ for acoustic waves, with $\beta=\beta_{i}+\beta_{e}$.

\section{Nonlinear equations for MHD waves in non-neutral plasmas}

The standard equations of continuity and momentum for a collisionless plasma are given by

$$
\begin{aligned}
& \partial_{t} N_{j}+\nabla \cdot\left(N_{j} \boldsymbol{V}_{j}\right)=0 \\
& N_{j} m_{j} \frac{\mathrm{d} \boldsymbol{V}_{j}}{\mathrm{~d} t}=N_{j} q_{j}\left(\boldsymbol{E}+\boldsymbol{V}_{j} \times \boldsymbol{B}\right)-\nabla \cdot \mathbb{P}_{j},
\end{aligned}
$$

where $N_{j}$ is density, $m_{j}$ mass, $q_{j}$ charge, $\boldsymbol{V}_{j}$ velocity and $\mathbb{P}_{j}$ pressure for species “ $j$ ”. Assuming a plasma of single charged ions and electrons $\left(q_{i}=-q_{e}=e\right)$, two linear combinations of electron and ion equations (e.g. Krall and Trivelpiece, 1973) give the plasma momentum equation,

$N m_{i} \frac{\mathrm{d} \boldsymbol{V}}{\mathrm{d} t}=\varepsilon_{0} \boldsymbol{E} \nabla \cdot \boldsymbol{E}+\boldsymbol{J} \times \boldsymbol{B}-\nabla \cdot \mathbb{P}$,

and the generalized Ohm's law,

$$
\begin{aligned}
& \frac{m_{e}}{N e^{2}}\left[\frac{\partial \boldsymbol{J}}{\partial t}+\nabla \cdot\left(\boldsymbol{V} \boldsymbol{J}+\boldsymbol{J} \boldsymbol{V}-\boldsymbol{V} \boldsymbol{V} \varepsilon_{0} \nabla \cdot \boldsymbol{E}-\boldsymbol{J} \boldsymbol{J} \frac{1}{e N}\right)\right] \\
& +\frac{1}{N e}\left(\boldsymbol{J} \times \boldsymbol{B}-\nabla \cdot \mathbb{P}_{e}\right)=\boldsymbol{E}+\boldsymbol{V} \times \boldsymbol{B},
\end{aligned}
$$

where $\boldsymbol{V}, \boldsymbol{J}$ and $\mathbb{P}$ are the center of mass velocity, current density and total pressure, respectively. As usual, the above equations are complemented by the Maxwell's equations

$\nabla \times \boldsymbol{B}-\frac{1}{c^{2}} \frac{\partial \boldsymbol{E}}{\partial t}=\mu_{0} \boldsymbol{J}, \quad \nabla \times \boldsymbol{E}=-\frac{\partial \boldsymbol{B}}{\partial t}$,

and the equations of state for the electron pressure $p_{e}$, and the total pressure $p=p_{e}+p_{i}$, that are both assumed to take the polytropic form

$p_{e}=p_{e 0} n^{\gamma_{e}}, p=p_{0} n^{\gamma}$,

applicable for cold, or warm plasmas; $\beta<1$. In deriving Eqs. (15) and (16), $m_{e} \ll m_{i}$ and $N_{e} \approx N_{i} \approx N$ have been assumed, except for the difference $N_{e}-N_{i}$, where Poisson's Eq. (5) has been utilized. The charge separation effects are important for perpendicular particle acceleration as seen in Eqs. (4-6). Furthermore, large electric potentials that can be created by alfvenons (Stasiewicz and Ekeberg, 2008) imply that charge separation effects may be important for these structures.

Assuming one-dimensional propagation along the $\mathrm{x}$ axis and introducing normalized variables $b_{y, z}=B_{y, z} / B_{0}$, $e_{x, y, z}=E_{x, y, z} /\left(V_{A} B_{0}\right)$ and $v_{x, y, z}=V_{x, y, z} / V_{A}$, we can write the continuity equation as

$\frac{\partial n}{\partial t}+\frac{\partial}{\partial x}\left(n v_{x}\right)=0$,

and the momentum Eq. (15) as

$n\left(\frac{\partial v_{x}}{\partial t}+v_{x} \frac{\partial v_{x}}{\partial x}\right)=-\frac{1}{2} \frac{\partial b^{2}}{\partial x}-\frac{\beta}{2} \frac{\partial n^{\gamma}}{\partial x}+R_{a} e_{x} \frac{\partial e_{x}}{\partial x}$

$n\left(\frac{\partial v_{y}}{\partial t}+v_{x} \frac{\partial v_{y}}{\partial x}\right)=\frac{\partial b_{y}}{\partial x} \cos \alpha+R_{a} e_{y} \frac{\partial e_{x}}{\partial x}$

$n\left(\frac{\partial v_{z}}{\partial t}+v_{x} \frac{\partial v_{z}}{\partial x}\right)=\frac{\partial b_{z}}{\partial x} \cos \alpha+R_{a} e_{z} \frac{\partial e_{x}}{\partial x}$.

The vector components of the generalized Ohm's law (Eq. 16) read

$\frac{1}{2 n} \frac{\partial}{\partial x} b^{2}+\frac{\beta_{e}}{2 n} \frac{\partial}{\partial x} n^{\gamma_{e}}+e_{x}+v_{y} b_{z}-v_{z} b_{y}=0$

$-\frac{R_{m}}{n} \frac{\partial}{\partial x}\left[\frac{\partial b_{z}}{\partial t}+v_{x} \frac{\partial b_{z}}{\partial x}\right]=$ 


$$
\begin{aligned}
& -\frac{\partial b_{y}}{\partial x} \frac{\cos \alpha}{n}+e_{y}+v_{z} \cos \alpha-v_{x} b_{z} \\
\frac{R_{m}}{n} & \frac{\partial}{\partial x}\left[\frac{\partial b_{y}}{\partial t}+v_{x} \frac{\partial b_{y}}{\partial x}\right]= \\
& -\frac{\partial b_{z}}{\partial x} \frac{\cos \alpha}{n}+e_{z}+v_{x} b_{y}-v_{y} \cos \alpha
\end{aligned}
$$

where we have neglected the displacement current and introduced small parameters: $R_{m}=m_{e} / m_{i}$, and $R_{a}=V_{A}^{2} / c^{2}=\omega_{c i}^{2} / \omega_{p i}^{2}$. Space and time are normalized with the ion inertial length $\lambda_{i}$ and $\omega_{c i}$, respectively. The charge separation effects are represented by terms proportional to $R_{a}$. The Hall MHD approximation is obtained by setting $R_{m}=0$ in the above equations, while ideal MHD corresponds to setting all derivatives to zero.

Changing reference system to the stationary wave frame moving at the wave speed $\omega / k$, the explicit time dependence is removed. In the wave frame, plasma is streaming initially with velocity $\left(v_{x 0}, 0,0\right)$. Defining the Mach number $M=\omega / k V_{A}=-v_{x 0}$, the continuity equation in the wave frame yields

$n v_{x}=n_{0} v_{x 0}=-M$

while the momentum equations with space charge effects, $R_{a}$, are

$2 M^{2} n^{-1}+b^{2}+\beta n^{\gamma}-R_{a} e_{x}^{2}=\mathrm{const}$

$-M v_{y}=\cos \alpha b_{y}+R_{a} e_{y} e_{x}$

$-M v_{z}=\cos \alpha\left(b_{z}-b_{z 0}\right)+R_{a} e_{z} e_{x}$

where $b_{z 0}=\sin \alpha$. The generalized Ohm's law gives

$$
\begin{aligned}
e_{x} & =v_{z} b_{y}-v_{y} b_{z}-\frac{1}{2 n} \frac{\partial}{\partial x} b^{2}-\frac{\beta_{e}}{2 n} \frac{\partial}{\partial x} n^{\gamma_{e}} \\
& \approx M b_{y} \tan \alpha-\frac{\beta_{e}}{2 n} \frac{\partial}{\partial x} n^{\gamma_{e}} \\
& \frac{\cos \alpha}{n} \frac{\partial b_{y}}{\partial x}-e_{y}-v_{z} \cos \alpha+v_{x} b_{z}= \\
-\frac{R_{m}}{n} & \frac{\partial}{\partial x}\left(\frac{M}{n} \frac{\partial b_{z}}{\partial x}\right) \\
& \frac{\cos \alpha}{n} \frac{\partial b_{z}}{\partial x}-e_{z}+v_{y} \cos \alpha-v_{x} b_{y}= \\
+ & \frac{R_{m}}{n} \frac{\partial}{\partial x}\left(\frac{M}{n} \frac{\partial b_{y}}{\partial x}\right) .
\end{aligned}
$$

The boundary conditions for the flow and Eq. (17) imply that $e_{y}=-M \sin \alpha, e_{z}=0$ in the stationary wave frame.

\section{Linear dispersion equations}

To find the dispersion equation and regions of nonlinear solutions of Eqs. (26-32), we linearize them around the background state, $n=1+\delta n, b_{y}=0+\delta b_{y}, b_{z}=b_{z 0}+\delta b_{z}$, and seek exponentially varying solutions $\propto \exp \left(k \lambda_{i} x\right)$. Introducing notation $K=k \lambda_{i}$, we obtain a general polarization matrix

$\mathbb{M}=\left(\begin{array}{cc}K M_{\|}, & -A_{R}+M_{\|}^{2} D_{M} \\ A_{R}+R_{a} M_{\|}^{2} \sin ^{2} \alpha, & K M_{\|}\end{array}\right)$

which fulfills $\mathbb{M} \boldsymbol{b}_{T}=\mathbf{0}$, where $\boldsymbol{b}_{T}=\left[\delta b_{y}, \delta b_{z}\right]$ is a column vector with transverse magnetic perturbations. Here, $A_{R}=A-R_{m} M_{\|}^{2} K^{2}, \quad A=M_{\|}^{2}-1$ and $D_{M}=\sin ^{2} \alpha /\left(M^{2}-\gamma \beta / 2\right)$. The determinant of $\mathbb{M}$ gives the dispersion relation

$$
\begin{gathered}
K^{2}\left[1-R_{m}\left(2 A-M_{\|}^{2} D_{M}+R_{a} M_{\|}^{2} \sin ^{2} \alpha\right)\right]= \\
-\left(A M_{\|}^{-2}-D_{M}\right)\left(A+R_{a} M_{\|}^{2} \sin ^{2} \alpha\right)-K^{4} M_{\|}^{2} R_{m}^{2},
\end{gathered}
$$

which describes waves in the following branches: Alfvén, kinetic Alfvén, electron inertial Alfvén, magnetosonic, acoustic, ion cyclotron, lower hybrid, and whistler waves. Retaining only the leading orders of the small parameters $R_{a}, R_{m}$, we obtain a simpler expression,

$$
k^{2} \lambda_{i}^{2}=\frac{\left(A M_{\|}^{-2}-D_{M}\right)\left(A+R_{a} M_{\|}^{2} \sin ^{2} \alpha\right)}{R_{m}\left(2 A-M_{\|}^{2} D_{M}\right)-1},
$$

which will be used in the following analysis. The wave frequencies can be expressed as (note that $\lambda_{i}=V_{A} / \omega_{c i}$ )

$\frac{\omega}{\omega_{c i}}=k \lambda_{i} M$,

where $\omega_{c i}$ is the ion gyrofrequency.

Exponentially varying nonlinear waves correspond to solutions of Eq. (35) with $k^{2}>0$, while the reverse condition applies to linear (sinusoidal) waves. There are also areas in the dispersion diagram corresponding to complex roots $K^{2}$ of the full Eq. (34), which describe oscillitons (Sauer et al., 2001; Dubinin et al., 2005).

We also note a controversy on the importance of charge separation effects for the existence of soliton solutions (evanescent waves) between Verheest $(2007 \mathrm{a}, \mathrm{b})$ and McKenzie et al. (2007). The charge separation terms with $R_{a}$ do not significantly modify the dispersion relation for nonrelativistic plasmas and are not necessary for solitary wave solutions. A conclusion of non-existence of solitary wave solutions in quasi-neutral plasmas (Verheest, 2007a) is based on the incorrect notion that the Poisson Eq. (5) implies $E_{x} \approx 0$ for $\delta N_{e} / N \approx 0$. In fact, $E_{x}$ is determined by the Ohm's law (Eqs. 16, 30) and can be large and variable, while Eq. (5) describes the spatial distribution of the space charge $\delta N_{e}$ associated with a given profile of $E_{x}(x)$.

\subsection{Dispersive Alfvén waves}

From Eq. (35) we find that a nondispersive Alfvén mode described by $A+R_{a} M_{\|}^{2} \sin ^{2} \alpha=0$ propagates with speed

$\frac{\omega^{2}}{k_{\|}^{2}}=\frac{V_{A}^{2}}{1+\frac{V_{A}^{2}}{c^{2}} \sin ^{2} \alpha}$, 
if space charge effects are retained. Close to the Alfvén mode, $M_{\|}^{2}=1$, the dispersion of sinusoidal waves can be found for all angles by a series expansion of Eq. (35) in the limit $R_{a} \approx 0$ as

$$
\frac{\omega^{2}}{k_{\|}^{2} V_{A}^{2}}=1+\frac{D}{2}-\frac{\operatorname{sgn}(D)}{2}\left(D^{2}+4 k^{2} \lambda_{i}^{2}+4 k^{2} \lambda_{e}^{2} D\right)^{1 / 2},
$$

where $k_{\|}=k \cos \alpha, \quad D=\sin ^{2} \alpha /\left(\cos ^{2} \alpha-\gamma \beta / 2\right)$. This is the equation for dispersive Alfvén waves (DAW) that include electron inertial Alfvén waves (EIAW), kinetic Alfvén waves (KAW), and shear Alfvén waves for arbitrary $\beta$ and propagation angle $\alpha$. For parallel propagation, Eq. (38) becomes

$$
\frac{\omega^{2}}{k_{\|}^{2} V_{A}^{2}} \approx 1-k \lambda_{i}
$$

while for larger angles satisfying $D^{2} \gg k^{2} \lambda_{i}^{2}$, a series expansion in $k^{2}$ gives

$$
\frac{\omega^{2}}{k_{\|}^{2} V_{A}^{2}} \approx 1+\frac{k^{2} \lambda_{i}^{2}}{\sin ^{2} \alpha}\left(\frac{\gamma \beta}{2}-\cos ^{2} \alpha\right)-k^{2} \lambda_{e}^{2} .
$$

For quasi-perpendicular propagation and $\gamma \beta / 2 \gg \cos ^{2} \alpha$, Eq. (40) reduces to a perpendicular dispersive mode

$$
\frac{\omega^{2}}{k_{\|}^{2} V_{A}^{2}} \approx 1+\frac{\gamma \beta}{2} k^{2} \lambda_{i}^{2}-k^{2} \lambda_{e}^{2}
$$

The transition between ion dominated dispersion and electron dispersion at $\beta \approx m_{e} / m_{i}$, implied by this equation, has been verified in laboratory experiments by Kletzing et al. (2003).

It is seen that the ion inertia completely dominates the dispersive properties of Alfvén waves and that electron inertia becomes significant only for $\beta \ll m_{e} / m_{i}$, and $\alpha>88.7^{\circ}$ where

$$
\frac{\omega^{2}}{k_{\|}^{2} V_{A}^{2}} \approx 1-k^{2} \lambda_{e}^{2} \approx \frac{1}{1+k^{2} \lambda_{e}^{2}},
$$

which is the equation for electron inertial Alfvén waves, EIAW.

We note that the ion gyroradius, $\rho_{i}=v_{t i} / \omega_{c i}$ is related to the ion beta $\beta_{i}=2 \mu_{0} p_{i} / B^{2}$ by the relation $\rho_{i}^{2}=\lambda_{i}^{2} \beta_{i}$. This implies that finite gyroradius effects of kinetic theory are incorporated as finite beta effects in fluid models. Indeed, assuming $\gamma \approx 2$ and neglecting electron inertia when $\beta>m_{e} / m_{i}$, we recover from Eq. (41) the well known dispersion relation for kinetic Alfvén waves (KAW),

$\frac{\omega^{2}}{k_{\|}^{2} V_{A}^{2}} \approx 1+\frac{\gamma}{2}\left(\beta_{i}+\beta_{e}\right) k^{2} \lambda_{i}^{2}=1+k_{\perp}^{2} \rho^{2}$,

where $\rho^{2}=\rho_{i}^{2}\left(1+T_{e} / T_{i}\right)=\rho_{s}^{2}\left(1+T_{i} / T_{e}\right)$, and $\rho_{s}$ is the acoustic gyroradius. Equation (43) corresponds to the formula derived by Hasegawa (1976) using kinetic theory, with a small difference in the expression for $\rho^{2}(3 / 4$ instead of 1$)$, see also Lysak and Lotko (1996).

Using Eqs. (30) and (43), we find that for quasiperpendicular KAW,

$\left|\frac{\delta E_{x}}{\delta B_{y}}\right| \approx V_{A} M_{\|} \sin \alpha \approx V_{A}\left(1+k_{\perp}^{2} \rho^{2}\right)^{1 / 2}$,

which is also a known result of kinetic theory.

\subsection{Dispersive magnetosonic waves}

We recall that the ideal (non-dispersive) Alfvén waves are obtained from the term $A=0$ in Eq. (35). Similarly, non-dispersive magnetosonic waves are described by $A M_{\|}^{-2}-D_{M}=0$ in the same equation. This gives the well known equation

$\frac{\omega_{ \pm}^{2}}{k^{2} V_{A}^{2}}=\frac{1}{2}\left(1+\frac{\gamma \beta}{2}\right) \pm \frac{1}{2}\left[\left(1+\frac{\gamma \beta}{2}\right)^{2}-2 \gamma \beta \cos ^{2} \alpha\right]^{1 / 2}$,

with minus - for slow, and plus + for fast magnetosonic waves. Expanding Eq. (35) around these modes, we obtain expressions for dispersive magnetosonic waves. In a low beta approximation, $\beta \lesssim 0.1$, the slow magnetosonic waves are described by

$\frac{\omega^{2}}{k^{2} V_{A}^{2}} \approx \frac{\gamma \beta}{2} \cos ^{2} \alpha\left(1-k^{2} \lambda_{i}^{2} \sin ^{2} \alpha\right)$.

In the same approximation, the equation for fast magnetosonic waves is

$\frac{\omega^{2}}{k^{2} V_{A}^{2}} \approx 1+\frac{\gamma \beta}{2} \sin ^{2} \alpha+k^{2} \lambda_{i}^{2}\left(\frac{\cos ^{2} \alpha}{\sin ^{2} \alpha}-R_{m}\right)$,

valid for $\alpha>0$. The slow wave has negative dispersion, while the fast wave changes dispersion from positive (ion inertia) to negative (electron inertia) at $\tan \alpha=\left(m_{i} / m_{e}\right)^{1 / 2}$.

\section{Dispersion diagrams}

In the preceding section, we have shown that the general dispersion Eq. (35) reproduces results of kinetic theory for EIAW and KAW. To explore the meaning of other solutions described by this equation, we use a method of dispersion diagrams introduced by Stasiewicz (2004a, 2005). Figure 1 shows such a diagram computed for a cold plasma, $\beta=10^{-5}$. The upper panel shows the logarithm of inverse wavelength $\lambda_{i} / \lambda=k \lambda_{i} / 2 \pi$. Magenta color marks the position of wavelengths $\lambda=\lambda_{i}$ and black color marks waves with $\lambda=\lambda_{e}$. Three vertical border lines correspond to the magnetosonic line (M) at the sound speed $V_{s}=(\gamma \beta / 2)^{1 / 2} V_{A}$, to the Alfvén mode (A-) at Alfvén Mach number 1, and to the whistler resonance speed

$$
\frac{\omega^{2}}{k^{2} V_{A}^{2}} \approx \frac{\cos ^{2} \alpha}{2 R_{m}}+\frac{1+\cos ^{2} \alpha}{2}+\frac{\gamma \beta}{2} R_{m} \tan ^{2} \alpha .
$$




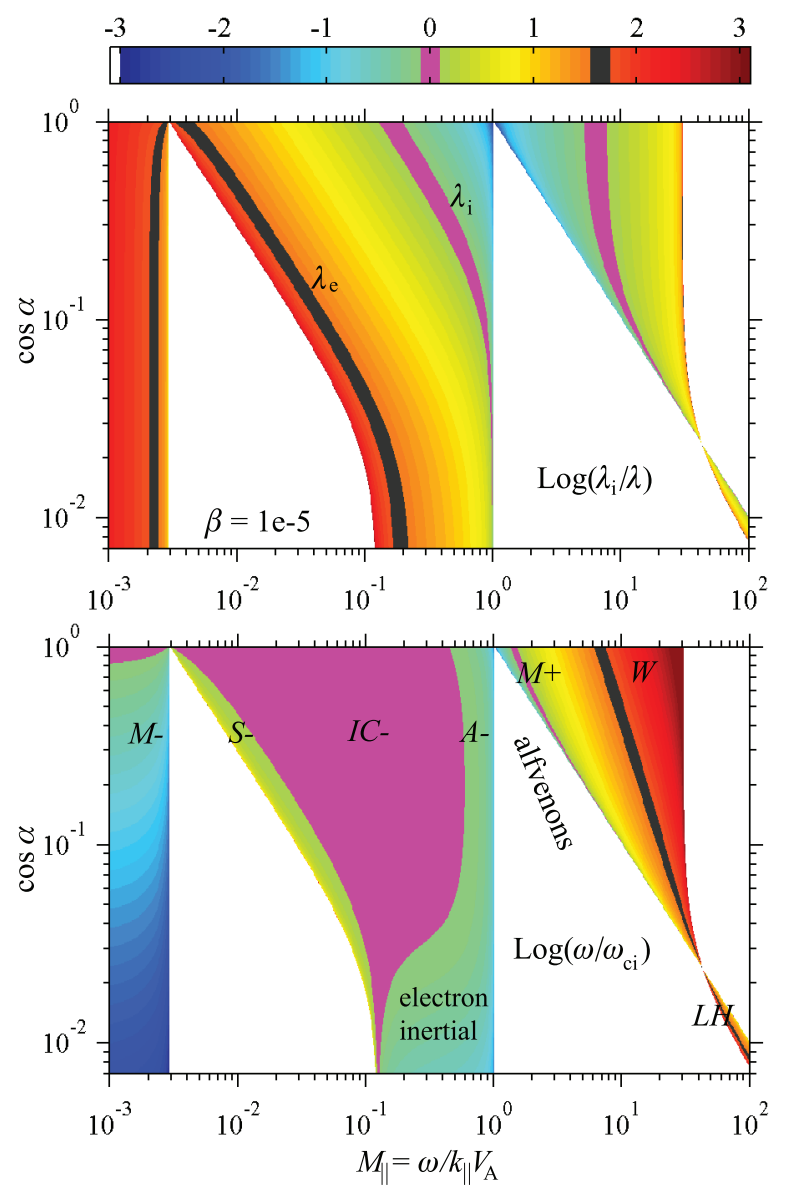

Fig. 1. Wave modes in a cold two-fluid plasma with $\beta=10^{-5}, \quad \gamma=5 / 3, \quad R_{m}=1 / 1836, \quad R_{a}=0$ obtained with Eq. (35). The upper part shows the color coded logarithm of inverse wavelength $\lambda_{i} / \lambda$ with marked wavelengths $\lambda_{i}$ (magenta), and $\lambda_{e}$ (black). The lower part shows the logarithm of frequency $\omega / \omega_{c i}$ with marked ion gyrofrequency $\omega_{c i}$ (magenta), and lower hybrid frequency $\omega_{L H}$ (black). White areas correspond to solitary wave solutions.

For parallel propagation, this speed is

$\frac{\omega^{2}}{k^{2}}=V_{W}^{2} \approx \frac{m_{i}}{2 m_{e}} V_{A}^{2}=\frac{p_{B}}{N m_{e}}$,

where $p_{B}=B^{2} / 2 \mu_{0}$ is the magnetic field pressure. By comparison to the sound speed, $V_{s}^{2}=\gamma p / N m_{i}$, and the Alfvén speed, $V_{A}^{2}=2 p_{B} / N m_{i}$, we see that the whistler wave speed (Eq. 49) can be interpreted as the magnetic sound carried by inertia of electron fluid with $\gamma_{e}=1$, while the Alfvén speed is the magnetic sound carried by ion fluid with $\gamma_{i}=2$.

The lower panel shows the logarithm of $\omega / \omega_{c i}$ given by Eq. (36) with marked positions of the ion gyrofrequency (magenta), and the lower hybrid frequency $\omega_{L H}=\left(\omega_{c i} \omega_{c e}\right)^{1 / 2}$ (black). One can identify the following cold plasma modes: slow magnetosonic (M-), acoustic (S-), ion cyclotron (IC-),

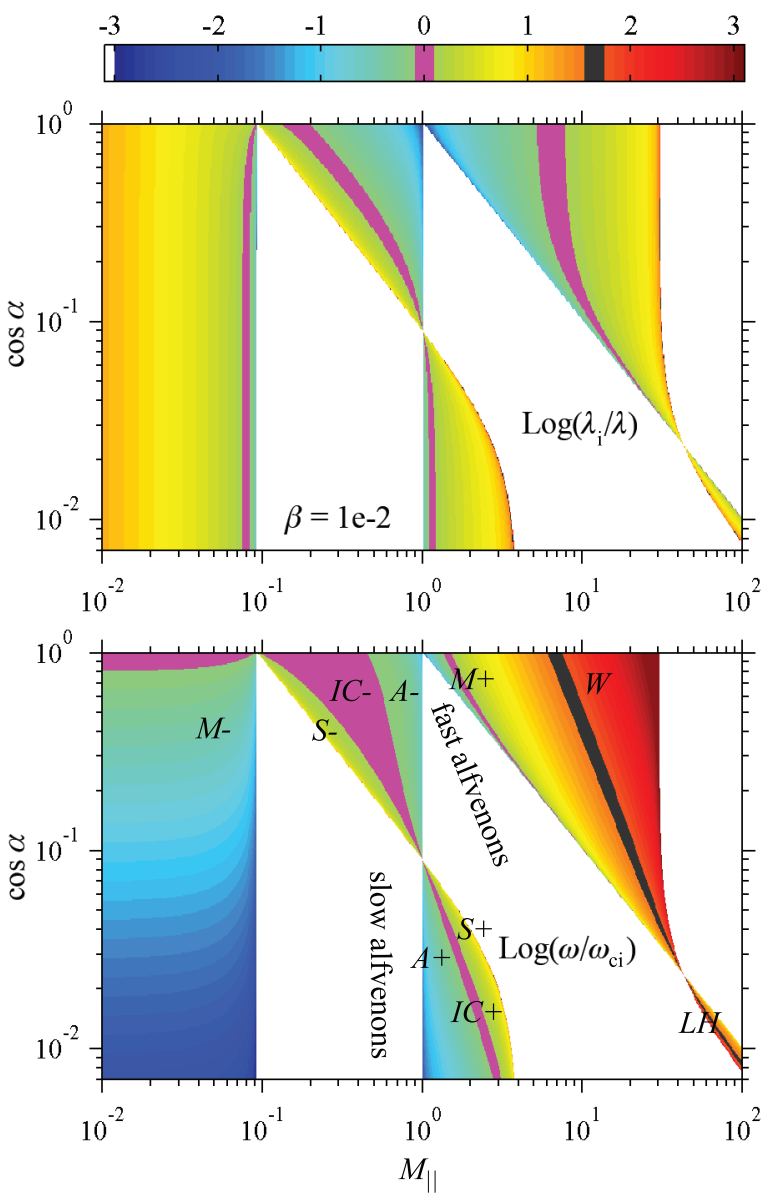

Fig. 2. Wave modes in a warm two-fluid plasma with $\beta=10^{-2}$ obtained with Eq. (35). Other parameters as in Fig. 1. The crossover position with kinetic modes $(\mathrm{A}+, \mathrm{IC}+\mathrm{S}+)$ is at $\cos ^{2} \alpha=\gamma \beta / 2$.

shear Alfvén (A-), fast magnetosonic (M+), lower hybrid $(\mathrm{LH})$, and whistler (W) modes. Signs "+" (for fast) and "-_" (for slow) refer to the parallel wave speeds $\omega / k_{\|}$with respect to the Alfvén speed $V_{A}$. White areas correspond to solitary wave solutions (alfvenons).

Alfvén waves at perpendicular phase directions become electron inertial Alfvén waves (EIAW), but they do not form a separate mode. Note that the commonly used term inertial Alfvén waves (IAW) for EIAW is misleading because it does not distinguish between electron and ion inertia. Electron inertia is significant only in the angular range $88.7-90^{\circ}$ and cold plasma, $\beta<m_{e} / m_{i}$, while ion inertia dominates at all other angles $0-88.7^{\circ}$ and all other beta, $\beta>m_{e} / m_{i}$.

Reducing plasma beta would move the magnetosonic Mand sound S-modes to the left of Fig. 1. Increasing beta would move these modes to the right. When $\beta \geq m_{e} / m_{i}$, the sound mode intersects with the Alfvén A- mode, and creates new kinetic modes shown in Fig. 2. Here we see that the sound mode intersects with the Alfvén line at 


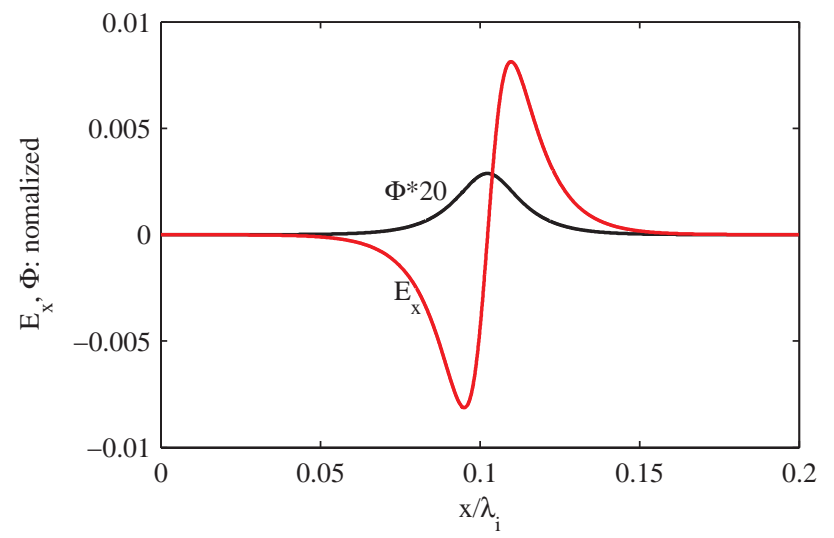

Fig. 3. Electric field, $E_{x} / V_{A} B_{0}$, and positive potential, $\Phi / \Phi_{A}$, for a slow alfvenon obtained by integration of nonlinear equations for $\beta=10^{-4}, \gamma=5 / 3, M=8 \times 10^{-4}, \cos \alpha=0.03$.

$\cos \alpha=V_{S} / V_{A}=(\gamma \beta / 2)^{1 / 2}$ creating the kinetic Alfvén wave, $(\mathrm{A}+)$, the fast perpendicular sound mode $(\mathrm{S}+)$, and the ion cyclotron mode (IC+). The parallel phase speed of these modes is faster than $V_{A}$, which justifies the + sign.

\section{Properties of alfvenons}

Solutions of Eqs. (26-32) in the nonlinear (white) regions of Figs. 1 and 2 represent solitary structures with bipolar electric fields that form either negative potential (convergent electric field $E_{x}$ ) or positive potential (divergent $E_{x}$ ). The electric potential along the wave path is

$\Phi(x)=-\int_{0}^{x} E_{x}\left(x^{\prime}\right) d x^{\prime}$.

Creation of a nonzero perpendicular electric potential results automatically in the establishment of a parallel potential drop of the same magnitude, and vice versa. This parallel potential can accelerate particles that move fast enough to leave the acceleration region before the wave changes polarisation. Numerical solutions of the nonlinear Eqs. (26-32) show that slow/fast alfvenons create positive/negative potentials, respectively. The sign of the potential is determined by the sign of $E_{x}$ in Eq. (10), which depends on the sense of polarization of the transverse magnetic field, or sign of $\delta B_{y}$. In the standard Runge-Kutta integration scheme we assume an initial perturbation $\delta b_{z}$ and other starting quantities are determined from the polarization matrix and the dispersion (34).

An example of a slow alfvenon is shown in Fig. 3. Figure 4 shows a series of two fast alfvenons that could correspond to multiple auroral arcs. The electric field is normalized with $V_{A} B_{0}$ and potential with $\Phi_{A}=V_{A} B_{0} \lambda_{i}$. Figure 4 is obtained directly from integration; it is not a composed picture. Each point in the white areas of Figs. 1 and 2 represents a solution which uniquely determines the size of the alfvenon and its physical properties such as: electric field,

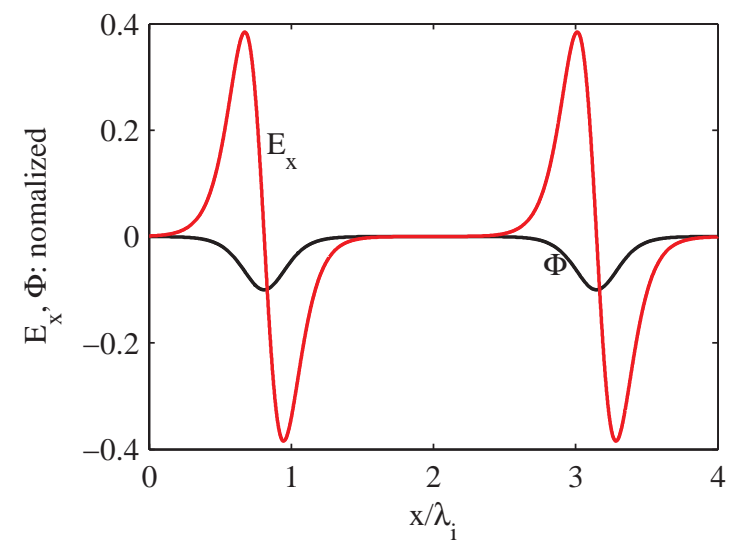

Fig. 4. A sequence of two fast alfvenons (convergent electric field structures) obtained by integration of nonlinear equations for $M=0.95, R_{m}=0$ (other parameters as in Fig. 3). Such alfvenons could correspond to multiple auroral arcs.

potential, magnetic field, currents and density perturbations. From the computed profiles we extract values for the maximum electric potential for alfvenons propagating at an arbitrary selected angle, $\cos \alpha=0.03$, shown in Fig. 5. It can be seen that fast alfvenons located between the Alfvén/acoustic modes and the fast magnetosonic mode can generate potentials that reach values $\Phi_{A}$. This potential goes down at the fast mode $M=1$. On the other hand, slow alfvenons, located between the KAW and the slow magnetosonic mode produce smaller potentials, which also become very small when approaching the slow mode. Note, however, that Fig. 5 computed for other propagation angles, or plasma beta, will give different values of $\Phi$. Generally, $\Phi \sim \Phi_{A}$ for alfvenons near the Alfvén mode, $M_{\|}=1$. Tables 1 and 2 show the characteristic potential $\Phi_{A}$ computed for plasma parameters above the auroral regions and in the solar corona, respectively. It can be seen that a single alfvenon can create potentials of $\sim 20 \mathrm{kV}$ in the aurora and hundreds of $\mathrm{kV}$ in the solar corona.

\section{Bursty ion flows as energy providers}

One of the energy sources available for acceleration of plasma both in the solar corona and in the magnetosphere is related to bursty ion flows $\delta V_{y}, \delta V_{z}$ that shake magnetic field lines $\delta B_{y}, \delta B_{z}$ and create parallel Poynting flux given by Eqs. (11) or (12). This flux is transported along the magnetic field lines and may convert to particle energy in far away regions. An example of bursty ion flows measured by Cluster in the magnetotail at position $(-16.7,-9.6,-1.8) R_{E}$ GSE (Geocentric Solar Ecliptic) is presented in Fig. 6. The ion flows are shown in the wave coordinate system used earlier in the theoretical analysis. The $\hat{x}$-axis is in the propagation direction determined as the minimum variance of $\mathbf{B}$. The $\hat{y}$-axis is defined as $\hat{\mathbf{y}}=-\hat{\mathbf{x}} \times \hat{\mathbf{b}}_{0}$, and $\hat{\mathbf{z}}$ completes the right 


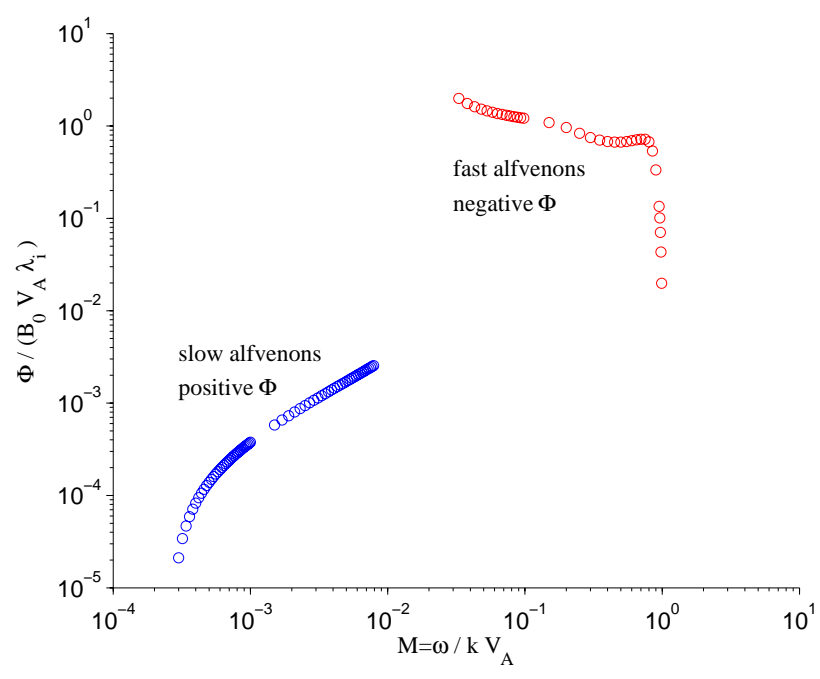

Fig. 5. Maximum electric potential in units of $\Phi_{A}=V_{A} B_{0} \lambda_{i}$ for solitons propagating at $\cos \alpha=0.03$ in a plasma with $\beta=10^{-4}$ and $\gamma=5 / 3$.

Table 1. Characteristic potential $\Phi_{A}=B_{0} V_{A} \lambda_{i}[\mathrm{kV}]$ for plasma conditions above the auroral region.

\begin{tabular}{rrrl}
\hline$N$ & & $B_{0}$ & {$[\mathrm{nT}]$} \\
{$\left[\mathrm{cm}^{-3}\right]$} & 1000 & 100 & 10 \\
\hline $10^{3}$ & 5 & 0.05 & \\
$10^{2}$ & 50 & 0.5 & \\
$10^{1}$ & 500 & 5 & 0.050 \\
$10^{0}$ & 5000 & 50 & 0.500 \\
\hline
\end{tabular}

handed system. The Poynting flux carried by Alfvén modes in Eq. (12) is

$S_{\|} \approx V_{A} N m_{i}\left\langle V_{y}\right\rangle^{2}$.

The ratio of the magnetic flux tube area in the magnetosphere and ionosphere is proportional to the ratio of the magnetic inductions: $A_{M} / A_{I}=B_{I} / B_{M}$. In effect, the Poynting flux (Eq. 51), scaled to ionospheric heights $\left(B_{I}=40 \mu \mathrm{T}\right)$, is independent of the magnetic field in the source, $B_{M}$, and equals $S_{\|} \approx 15 \mathrm{~mW} \mathrm{~m}^{-2}$ for proton plasma with $N \approx 0.1 \mathrm{~cm}^{-3}$ and flow velocity $V_{y} \approx 200 \mathrm{~km} \mathrm{~s}^{-1}$. Thus, the Poynting flux generated by ions shaking magnetic field lines at $\delta V_{y} \approx 100-600 \mathrm{~km} / \mathrm{s}$ in the distant magnetosphere, and transported by Alfvén waves toward the ionosphere, can explain the kinetic energy flux carried by accelerated auroral electrons, $S_{e} \approx 1-100 \mathrm{~mW} \mathrm{~m}^{-2}$, as measured by satellites.

The electric potential $\Phi$ created by alfvenons modifies particle trajectories in phase space. Conservation of the total energy, $m v_{\perp}^{2} / 2+m v_{\|}^{2} / 2+q \Phi+m \Phi_{G}=$ const, where $\Phi_{G}$ is the gravitational potential, implies that particles entering/leaving

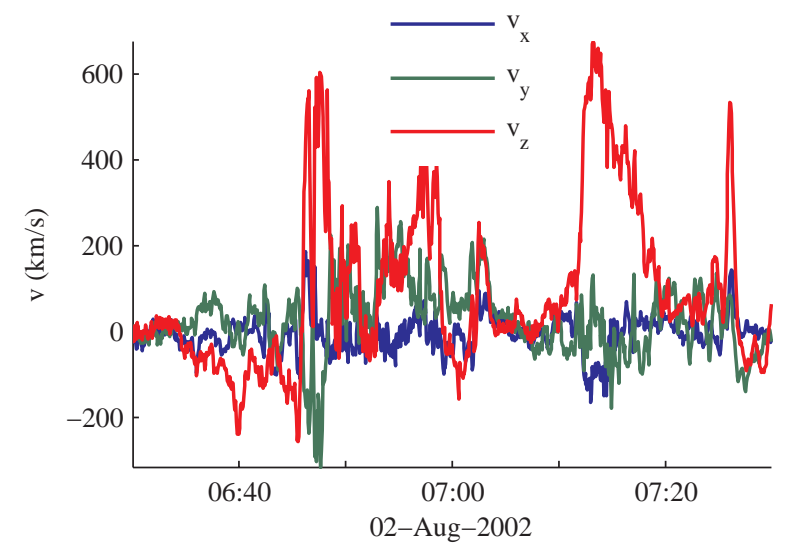

Fig. 6. An example of bursty ion flows measured by Cluster in the magnetotail at $(-16.7,-9.6,-1.8) R_{E}$ GSE (geocentric solar ecliptic) coordinates. These flows can produce magnetosonic and Alfvén waves carrying Poynting flux toward the ionosphere with power sufficient to drive auroral phenomena.

Table 2. Characteristic potential $\Phi_{A}[\mathrm{kV}]$ for plasma conditions in the solar corona.

\begin{tabular}{rrrll}
\hline$N$ & & $B_{0}$ & {$[\mathrm{G}]$} & \\
{$\left[\mathrm{cm}^{-3}\right]$} & 1000 & 100 & 10 & 1 \\
\hline $10^{11}$ & 500 & 5 & 0.05 & \\
$10^{10}$ & 5000 & 50 & 0.5 & \\
$10^{09}$ & & 500 & 5 & 0.05 \\
$10^{08}$ & & 5000 & 50 & 0.5 \\
\hline
\end{tabular}

the structure would change their kinetic energy by an amount corresponding to the difference of the potential energies. In a collisionless plasma the perpendicular velocity is constrained by conservation of the first adiabatic invariant, leading to an increase of $v_{\|}^{2}$ and lowering of the mirroring altitude of particles to denser regions where they can be subject to collisions. Particles will dissipate kinetic energy by instabilities related to pitch angle anisotropy, through X-ray emissions, heating of the ambient plasma, and ionization of the neutral atmosphere at low altitudes. Heating can also be accomplished by ion Landau damping of slow modes and electron Landau damping of fast modes. The perception of quasi-static auroral structures could apply to alfvenons that reached the collisional ionosphere, and through the retardation caused by the heavy load of plasma, dissipate the electromagnetic energy flux. The dissipative processes are beyond the formalism of the present stationary model and their description would require a fully kinetic model supported by simulations. 


\section{Alfvenons in the solar corona}

The problem of acceleration of plasma by alfvenons in the solar corona has been addressed recently by Stasiewicz (2006) and Stasiewicz and Ekeberg (2008). They found that fast alfvenons generated during magnetic reconfigurations in coronal loops can accelerate electrons to hundreds of keV, producing X-rays of solar flares. Furthermore, slow alfvenons driven by chromospheric convection $\left(\delta V_{y}, \delta V_{z}\right.$ drivers) would produce positive electric potentials that expel solar wind ions. The generation of solar wind with protons streaming at $300-800 \mathrm{~km} / \mathrm{s}$, after acquiring first the gravitational escape speed of $600 \mathrm{~km} / \mathrm{s}$, implies the presence of positive acceleration potentials, $2-5 \mathrm{kV}$ at the base of the corona. Scattering a fraction $(\sim 15 \%$, or $\sim 0.5 \mathrm{keV})$ of the ion kinetic energy by Coulomb collisions, cyclotron, or two-stream instabilities would produce particles with high coronal temperatures of $\sim 5 \mathrm{MK}$. Thus, the highly publicized problem of heating of the solar corona in fact reduces to the problem of cooling of particles accelerated by alfvenons.

The perpendicular size of alfvenons is a few ion inertial lengths, a tiny scale in the solar corona; $\lambda_{i}=20 \mathrm{~m}$ for $N=10^{8} \mathrm{~cm}^{-3}$. Because of the scaling properties, $\lambda_{i} \propto N^{-1 / 2}$, the alfvenons in the solar corona at $N \sim 10^{8}-10^{9} \mathrm{~cm}^{-3}$ are expected to be $10^{3}-10^{4}$ times thinner than the corresponding structures measured in the magnetosphere at $N \sim 10^{1}-10^{2} \mathrm{~cm}^{-3}$. However, the transverse horizontal extension (y-direction) would match the driver size of solar convection granulation, and the accelerated plasma will spread along $\mathbf{B}$; both dimensions are of many $\mathrm{Mm}$. Thin threads and arcades of heated plasma seen in images from the TRACE spacecraft (http://trace.lmsal.com) and Hinode (Cirtain et al., 2007) are possibly manifestations of remnants of alfvenons described above.

\section{Other theoretical models for dispersive Alfvén waves and solitons}

Solitons in the magnetoplasma were studied theoretically using Derivative Nonlinear Schrödinger (DNSL) equation by several authors (Hada et al., 1989; Mjølhus and Wyller, 1988; Spangler, 1990; Medvedev and Diamond, 1996; Nocera and Buti, 1996; Buti, 1999; Passot et al., 2005) The solutions concerned mainly circularly polarized waves propagating parallel to the magnetic field.

Another development concerned quasi-perpendicular propagation of dispersive Alfvén waves and processes leading to establishment of filamentary structures with small size perpendicular to B (e.g. Knudsen, 1996; Hollweg, 1999; Seyler and Lysak, 1999; Wu, 2003). Many models are based on restrictive assumption of linear magnetic polarization $\delta B_{y}\left(\delta B_{x}=\delta B_{z}=0\right)$, or that electrons move only parallel to the magnetic field, while ions only perpendicular. This could lead to incorrect conclusions reached by Seyler and Lysak (1999) on nonexistence of solitary wave solutions.

In the ionospheric Alfvén resonator region, where the Alfvén speed is strongly inhomogeneous, a nonlocal kinetic approach must be used to describe the wave-particle interaction according to Lysak and Song (2005). They combined kinetic and fluid effects in a hybrid approach, which describes the evolution of both the electron distribution and the fields. Their calculations suggest that, on average, $10 \%$ of the Poynting flux incident through Alfvén waves contribute to ionospheric precipitation and that the flux arrives in bursts once per wave period. It should also be mentioned that Song and Lysak (1994) used the term "Alfvenon" for a reconnection structure at the magnetopause. The alfvenons discussed in this paper have no relation to those reconnection structures, but instead represent soliton solutions located between the Alfvén and magnetosonic wave modes in dispersion diagrams such as those in Fig. 2.

Applications of kinetic Alfvén waves to problems of coronal heating were studied extensively by Voitenko and Goossens $(2004,2006)$. They showed that perpendicular ion heating can be produced as a result of interactions between ions and short wavelength KAW. These authors also proposed a mechanism for a resonant decay of shear Alfvén waves into two KAW (Voitenko and Goossens, 2005).

Recent development was stimulated by identification of magnetosonic solitons in Cluster measurements (Stasiewicz et al., 2003; Stasiewicz, 2004b), and modelling their properties with Hall-MHD equations integrated directly in the approach based on constants of motion (McKenzie et al., 2004), rather than on approximative DNSL solutions. Multi-fluid equations for bi-ion plasmas have oscillatory solutions with a soliton envelope, called "oscillitons" by Sauer et al. (2001). Existence and properties of solitons and oscillitons in a warm proton-alpha particle plasma have been studied further by Dubinin et al. (2003) and Dubinin et al. (2005).

\section{Summary}

By analyzing the two-fluid equations, we have found that nonlinear solutions, here called alfvenons, can provide explanations to several unsolved problems related to acceleration of plasma in the magnetosphere and the solar corona.

1. Alfvenons create bipolar electric field structures representing either negative potentials (convergent electric field), or positive potentials (divergent electric field) on spatial scales ranging from several ion inertial lengths to the electron inertial length. Structures on ion inertial scales $(\sim 50 \mathrm{~km})$ could correspond to auroral forms related to inverted-V type electron precipitation, while those on electron inertial scales could correspond to thin auroral $\operatorname{arcs}(\sim 1 \mathrm{~km})$. 
2. Since the alfvenons are produced naturally as trains of solitons (see Fig. 4), the model can also explain multiple arc systems frequently observed in aurora.

3. Negative electric potentials created by fast alfvenons could explain acceleration of auroral electrons to tens of $\mathrm{keV}$, and acceleration of electrons in the solar corona to hundreds of $\mathrm{keV}$.

4. Positive electric potentials created by slow alfvenons in the solar corona can lead to outflow and acceleration of solar wind ions to velocities of $300-800 \mathrm{~km} / \mathrm{s}$.

5. The parallel Poynting flux required to drive auroral processes can be provided by bursty bulk flows in the magnetotail. In the solar corona, the required energy flux can be provided by chromospheric convection granulation and by varying magnetic fields in coronal loops.

6. Space charge effects introduce terms proportional to $R_{a}=V_{A}^{2} / c^{2}$, which are negligible in non-relativistic plasmas and do not affect the existence of soliton solutions.

Acknowledgements. J. Ekeberg is financed by the Swedish National Graduate School of Space Technology.

Edited by: A. C. L. Chian

Reviewed by: two anonymous referees

\section{References}

Aschwanden, M.: Physics of the solar corona, Springer, 2004.

Balikhin, M., Gedalin, M., and Petrukovich, A.: New mechanism for electron heating in shocks, Phys. Rev. Lett., 70, 1259-1262, 1993.

Banerjee, D., Erdelyi, R., Oliver, R., and O'Shea, E.: Present and Future Observing Trends in Atmospheric Magnetoseismology, Solar Phys., 246, 3-29, 2007.

Buti, B.: Chaos in Magnetoplasmas, Nonlin. Processes Geophys., 6, 129-143, 1999, http://www.nonlin-processes-geophys.net/6/129/1999/.

Carlson, C. W., McFadden, J. P., Ergun, R. E., et al.: FAST observations in the downward auroral current region, Geophys. Res. Lett., 25, 2017-2020, 1998a.

Carlson, C. W., Pfaff, R. F., and Watzin, J. G.: The fast auroral snapshot mission, Geophys. Res. Lett., 25, 2013-2016, 1998 b.

Charles, C.: A review of recent double layer experiments, Plasma Sources Sci. Technol., 16, R1-R15, 2007.

Chaston, C. C., Peticolas, L. M., Bonnell, J. W., Carlson, C. W., Ergun, R. E., McFadden, J. P., and Strangeway, R. J.: Width and brightness of auroral arcs driven by inertial Alfvén waves, J. Geophys. Res., 108, 1091, doi:10.1029/2001JA007537, 2003.

Chaston, C. C., Peticolas, L. M., Carlson, C. W., et al.: Energy deposition by Alfvén waves into the dayside auroral oval: Cluster and FAST observations, J. Geophys. Res., 110, 2211, doi: 10.1029/2004JA010483, 2005.
Cirtain, J. W., Golub, L., Lundquist, L., et al.: Evidence for Alfvén Waves in Solar X-ray Jets, Science, 318, 1580, doi: 10.1126/science.1147050, 2007.

Cole, K. D.: Effects of crossed magnetic and spatially dependent electric fields on charged particle motion, Planet. Space Sci., 24, 515-518, 1976.

Cranmer, S. R., Field, G. B., and Kohl, J. L.: Spectroscopic constraints on models of ion cyclotron resonance heating in the polar solar corona, Astrophys. J., 518, 937-947, 1999.

DeMoortel, I., Ireland, J., and Walsh, R. W.: Observation of oscillations in coronal loops, Astr. Astrophys., 355, L23-26, 2000.

Dubinin, E., Sauer, K., McKenzie, J. F., and Chanteur, G.: Solitons, oscillitons, and stationary waves in a warm p-alpha plasma, J. Geophys. Res., 108, 1296, doi:10.1029/2002JA009572, 2003.

Dubinin, E., Sauer, K., and McKenzie, J. F.: Nonlinear inertial and kinetic Alfvén waves, J. Geophys. Res., 110, A10S04, doi:10. 1029/2004JA010770, 2005.

Ergun, R. E., Carlson, C. W., McFadden, J. P., et al.: FAST satellite observations of electric field structures in the auroral zone, Geophys. Res. Lett., 25, 2025-2028, 1998.

Gekelman, W., Vincena, S., Palmer, N., Pribyl, P., Leneman, D., Mitchell, C., and Maggs, J.: Experimental measurements of the propagation of large-amplitude shear Alfvén waves, Plasma Phys. Contr. Fusion, 42, B15-B26, 2000.

Gekelman, W., Maggs, J., Morales, G., et al.: Basic plasma science facility, http://plasma.physics.ucla.edu/bapsf, 2008.

Gurnett, D. A.: Electric Field and Plasma Observations in the Magnetosphere, in: Critical Problems of Magnetospheric Physics, edited by: Dyer, E. R., pp. 123-138, AGU, 1972.

Hada, T., Kennel, C. F., and Buti, B.: Stationary nonlinear Alfvén waves and solitons, J. Geophys. Res., 94, 65-77, 1989.

Hasegawa, A.: Particle acceleration by MHD surface wave and formation of aurora, J. Geophys. Res., 81, 5083-5090, 1976.

Hollweg, J. V.: Kinetic Alfvén wave revisited, J. Geophys. Res., 104, 14 811-14 819, 1999.

Ivchenko, N. and Marklund, G.: Current singularities observed on Astrid-2, Adv. Space Res., 30, 1779-1782, 2002.

Janhunen, P., Olsson, A., Russell, C. T., and Laakso, H.: Alfvénic electron acceleration in aurora occurs in global Alfvén resonosphere region, Space Sci. Rev., 122, 89-95, doi:10.1007/ s11214-006-7017-5, 2006.

Keiling, A., Wygant, J. R., Cattell, C., Temerin, M., Mozer, F. S., Kletzing, C. A., Scudder, J., Russell, C. T., Lotko, W., and Streltsov, A. V.: Large Alfvén wave power in the plasma sheet boundary layer during the expansion phase of substorms, Geophys. Res. Lett., 27, 3169-3172, doi:10.1029/2000GL000127, 2000.

Keiling, A., Wygant, J. R., Cattell, C., Peria, W., Parks, G., Temerin, M., Mozer, F. S., Russell, C. T., and Kletzing, C. A.: Correlation of Alfvén wave Poynting flux in the plasma sheet at 4-7 Re with ionospheric energy flux, J. Geophys. Res., 107, 1132, doi:10.1029/2001JA900140, 2002.

Kletzing, C. A., Bounds, S. R., Martin-Hiner, J., Gekelman, W., and Mitchell, C.: Measurements of the shear Alfvén wave dispersion for finite perpendicular wave number, Phys. Rev. Lett., 90, 035 004, doi:10.1103/PhysRevLett.90.035004, 2003.

Knudsen, D. J.: Spatial modulation of electron energy and density by nonlinear stationary inertial Alfvén waves, J. Geophys. Res., 101, 10 761-10 772, doi:10.1029/96JA00429, 1996. 
Kohl, J. L., Noci, G., Antonucci, E., et al.: First results from the Soho ultraviolet coronograph spectrometer, Solar Phys., 175, 613-644, 1997.

Krall, N. A. and Trivelpiece, A. W.: Principles of Plasma Physics, McGraw-Hill, 1973.

Louarn, P., Wahlund, J. E., Chust, T., de Feraudy, H., Roux, A., Holback, B., Dovner, P. O., Eriksson, A. I., and Holmgren, G.: Observation of kinetic Alfvén waves by the Freja spacecraft, Geophys. Res. Lett., 21, 1847-1850, 1994.

Lundin, R., Haerendel, G., and Grahn, S.: The Freja mission, J. Geophys. Res., 103, 4119-4123, 1998.

Lysak, R. L. and Lotko, W.: On the kinetic dispersion equation for shear Alfvén waves, J. Geophys. Res., 101, 5085-5094, 1996.

Lysak, R. L. and Song, Y.: Nonlocal interactions between electrons and Alfvén waves on auroral field lines, J. Geophys. Res., 110, 10, doi:10.1029/2004JA010803, 2005.

Marklund, G. T., Blomberg, L. G., Fälthammar, C.-G., and Lindqvist, P.-A.: On intense diverging electric fields associated with black aurora, Geophys. Res. Lett., 21, 1859-1862, 1994.

Marklund, G. T., Ivchenko, N., Karlsson, T., et al.: Temporal evolution of the electric field accelerating electrons away from the auroral ionosphere, Nature, 414, 724-727, 2001.

McKenzie, J. F., Dubinin, E., Sauer, K., and Doyle, T. B.: The applications of the constants of motion to nonlinear stationary waves in complex plasmas: a unified fluid dynamic viewpoint, J. Plasma Phys., 70, 431, doi:10.1017/S0022377803002654, 2004.

McKenzie, J. F., Dubinin, E., and Sauer, K.: Comment on "Obliquely propagating large amplitude solitary waves in charge neutral plasmas" by F. Verheest, Nonlin. Processes Geophys., 14, 4957, 2007, Nonlin. Processes Geophys., 14, 543-544, 2007, http://www.nonlin-processes-geophys.net/14/543/2007/.

Medvedev, M. V. and Diamond, P. H.: Fluid models of kinetic effects on coherent nonlinear Alfvén waves, Phys. Plasmas, 3, 863, doi:10.1063/1.871790, 1996.

Mishin, E. and Banaszkiewicz, M.: On auroral ion conics and electron beam acceleration, Geophys. Res. Lett., 25, 4309-4312, 1998.

Mjølhus, E. and Wyller, J.: Nonlinear Alfvén waves in a finite beta plasma, J. Plasma Phys., 40, 299-318, 1988.

Mozer, F. S., Cattell, C. A., Hudson, M. K., Lysak, R. L., Temerin, M., and Torbert, R. B.: Satellite measurements and theories of low altitude auroral particle acceleration, Space Sci. Rev., 27, 155-213, 1980.

Nocera, N. and Buti, B.: Acceleration of Alfvén solitons, Physica Scripta, T63, 186, 1996

Palmer, N., Gekelman, W., and Vincena, S.: Measurement of ion motion in a shear Alfvén wave, Phys. Plasmas, 12, 2102, doi: 10.1063/1.1930796, 2005.

Paschmann, G., Haaland, S., Treumann, R., et al.: Auroral plasma physics, Space Sci. Rev., 103, 1-500, 2002.

Passot, T., Sulem, C., and Sulem, P. L.: Linear versus nonlinear dissipation for critical NLS equation, Physica D, 203, 167, doi:10.1016/j.physd.2005.03.011, 2005.

Robbrecht, E., Verwichte, E., Berghamans, D., Hochedez, J. F., Poedts, S., and Nakariakov, V. M.: Slow magnetoacoustic waves in coronal loops: EIT and TRACE, Astr. Astrophys., 370, 591, doi:10.1051/0004-6361:20010226, 2001.

Sakao, T., Kano, R., Narukage, N., et al.: Continuous plasma outflows from the edge of solar active region as a possible source of solar wind, Science, 318, 1585, doi:10.1126/science.114792, 2007.

Samoznaev, L. N., Efimov, A. I., Andreev, V. E., Chashei, I. V., and Bird, M. K.: Properties of Alfvén waves in the outer solar corona from two-station Faraday rotation observations, Phys. Chem. Earth, 25, 107-112, 2000.

Sauer, K., Dubinin, E., and McKenzie, J.: New type of soliton in biion plasmas and possible implications, Geophys. Res. Lett., 28, 3589-3592, 2001.

Sauer, K., Dubinin, E., and McKenzie, J. F.: Solitons and oscillitons in multi-ion space plasmas, Nonlin. Processes Geophys., 10, 121-130, 2003, http://www.nonlin-processes-geophys.net/10/121/2003/.

Seyler, C. E. and Lysak, R. L.: On the existence of alfvénic solitary waves, Phys. Plasmas, 6, 4778, doi:10.1063/1.873766, 1999.

Song, Y. and Lysak, R. L.: Alfvénon, driven reconnection and the direct generation of the field-aligned current, Geophys. Res. Lett., 21, 1755-1758, 1994.

Spangler, S. R.: Kinetic effects of Alfvén wave nonlinearity, Phys. Fluids, B2, 407, doi:10.1063/1.859329, 1990.

Stasiewicz, K.: Theory and observations of slow-mode solitons in space plasmas, Phys. Rev. Lett., 93, 125 004, doi:10.1103/ PhysRevLett.93.125004, 2004a.

Stasiewicz, K.: Reinterpretation of mirror modes as trains of slow magnetosonic solitons, Geophys. Res. Lett., 31, L21 804, doi: 10.1029/2004GL021282, 2004b.

Stasiewicz, K.: Nonlinear Alfvén, magnetosonic, sound, and electron inertial waves in fluid formalism, J. Geophys. Res., 110, A03 220, doi:10.1029/2004JA010852, 2005.

Stasiewicz, K.: Heating of the solar corona by dissipative Alfvén solitons, Phys. Rev. Lett., 96, 175 003, doi:10.1103/PhysRevLett. 96.175003, 2006.

Stasiewicz, K.: Acceleration of particles in space plasmas by nonlinear magnetosonic waves, Plasma Phys. Contr. Fussion., 49, B621-B628, doi:10.1088/0741-3335/49/12B/S58, 2007.

Stasiewicz, K. and Ekeberg, J.: Electric potentials and energy fluxes available for particle acceleration by alfvenons in the solar corona, ApJL, 680, L153-L156, 2008.

Stasiewicz, K., Gustafsson, G., Marklund, G., Lindqvist, P.-A., Clemmons, J. H., and Zanetti, L.: Cavity resonators and Alfvén resonance cones observed on Freja, J. Geophys. Res., 102, 25652575, 1997.

Stasiewicz, K., Holmgren, G., and Zanetti, L.: Density depletions and current singularities observed by Freja, J. Geophys. Res., 103, 4251-4260, 1998.

Stasiewicz, K., Bellan, P., Chaston, C., Kletzing, C., Lysak, R., Maggs, J., Pokhotelov, O., Seyler, C., Shukla, P., Stenflo, L., Streltsov, A., and Wahlund, J.-E.: Small scale Alfvénic structure in the aurora, Space Sci. Rev., 92, 423-533, 2000a.

Stasiewicz, K., Lundin, R., and Marklund, G.: Stochastic ion heating by orbit chaotization on electrostatic waves and nonlinear structures, Phys. Scripta, T84, 60-63, 2000b.

Stasiewicz, K., Shukla, P. K., Gustafsson, G., Buchert, S., Lavraud, B., Thide, B., and Klos, Z.: Slow magnetosonic solitons detected by the Cluster spacecraft, Phys. Rev. Lett., 90, 085 002, doi:10. 1103/PhysRevLett.90.085002, 2003.

Tsiklauri, D.: A mechanism for parallel electric field generation in the MHD limit, A and A, 455, 1073, doi:10.1051/00046361:20064816, 2006. 
Verheest, F.: Obliquely propagating large amplitude solitary waves in charge neutral plasmas, Nonlin. Processes Geophys., 14, 4957, 2007a, http://www.nonlin-processes-geophys.net/14/49/2007/.

Verheest, F.: Reply to J. F. McKenzie et al.'s comment on "Obliquely propagating large amplitude solitary waves in charge neutral plasmas”, Nonlin. Processes Geophys., 14, 545-546, $2007 \mathrm{~b}$ http://www.nonlin-processes-geophys.net/14/545/2007/.

Vincena, S., Gekelman, W., and Maggs, J.: Shear Alfvén wave perpendicular propagation from the kinetic to the inertial regime, Phys. Rev. Lett., 93, 105003, doi:10.1103/PhysRevLett93. 105003, 2004.

Voitenko, Y. and Goossens, M.: Cross-field heating of coronal ions by low-frequency kinetic Alfvén waves, ApJL, 605, L149, doi:10.1086/420927, 2004.

Voitenko, Y. and Goossens, M.: Cross-Scale Nonlinear Coupling and Plasma Energization by Alfvén Waves, Phys. Rev. Lett., 94, 135 003, doi:10.1103/PhysRevLett.94.135003, 2005.
Voitenko, Y. and Goossens, M.: Energization of plasma species by intermittent kinetic Alfvén waves, Space Sci. Rev., 122, 255270, 2006.

Wahlund, J.-E., Louarn, P., Chust, T., de Feraudy, H., Roux, A., Holback, B., Dovner, P.-O., and Holmgren, G.: On ion accoustic turbulence and the nonlinear evolution of kinetic Alfvén waves in aurora, Geophys. Res. Lett., 21, 1831-1834, 1994.

Wu, D. J.: Dissipative solitary kinetic Alfvén wave and electron acceleration, Phys. Plasmas, 10, 1364, doi:10.1063/1.1564821, 2003.

Wygant, J. R., Keiling, A., Cattell, C. A., et al.: Polar spacecraft based comparisons of intense electric fields and Poynting flux near and within plasma sheet-tail lobe boundary to UVI images., J. Geophys. Res., 105, 18 675-18692, 2000. 